\title{
Results of Rectangular and Triangular Shaped Scleral Flaps in Trabeculectomy in Terms of Control of Intra Ocular Pressure (IOP) and Complications
}

\author{
Qirat Qurban ${ }^{1}$, Zeeshan Kamil ${ }^{2}$, Khalid Mahmood ${ }^{3}$ \\ ${ }^{1-3}$ Khalid Eye Clinic, Nazimabad Karachi
}

\begin{abstract}
Purpose: To compare between the results of rectangular and triangular shaped scleral flaps in trabeculectomy for primary open angle glaucoma.

Study design: Quasi experimental study

Place and Duration of Study: Khalid eye clinic, Karachi, from July 2018 to June 2019.

Methods: This study included 24 patients of either gender and age from 40 to 65 years. Patients with a diagnosis of primary open angle glaucoma refractory to medical treatment were included by convenient sampling technique. They were divided into two groups. Group A underwent trabeculectomy with rectangular shaped scleral flap whereas Group B underwent trabeculectomy with triangular shaped scleral flap. The main outcome measures were intraocular pressure reduction, anterior chamber depth and post-operative complications. All patients were followed up for a period of two months.
\end{abstract}

Results: Mean age was $52.1 \pm 6.72$ years. Mean pre-operative intraocular pressure in group A was $24.4 \pm 2.13$ $\mathrm{mmHg}$ and $23.5 \pm 1.64 \mathrm{mmHg}$ in group B. Mean post-operative IOP was $11.6 \pm 1.51 \mathrm{mmHg}$ in group $A$ and $13.4 \pm 1.67 \mathrm{mmHg}$ in group $B$ with $p$-value of 0.11 . Pre-operative anterior chamber depth (ACD) was grade 4 both groups. It remained same in all patients of group A but two patients of group B changed to grade 3 ACD. Two patients of group $B$ developed hypotony on the first post-operative day. They did not require revision suturing and were managed conservatively.

Conclusion: Both types of scleral flap are effective in reducing IOP but rectangular scleral flap reduced IOP more as compared to triangular scleral flap but the difference was not statistically significant.

Keywords: Trabeculectomy, Intra ocular pressure, Open angle glaucoma.

How to Cite this Article: Qurban Q, Kamal Z, Mahmood K. Comparison of Rectangular and Triangular Shaped Scleral Flaps in Trabeculectomy in Terms of Control of Intra Ocular Pressure (IOP) and Complications. Pak J Ophthalmol. 2021, 37 (4): 375-378.

Doi: $10.36351 /$ pjo.v37i3.1089

\section{INTRODUCTION}

Trabeculectomy is considered as the gold standard surgical technique for medically uncontrolled

Correspondence: Qirat Qurban

Khalid Eye Clinic, Nazimabad Karachi

Email: Khalid Eye Clinic, Nazimabad Karachi

Received: June 28, 2020

Revised: January 2021

Accepted: June 07, 2021 through which aqueous humor can traverse within the sclera from the anterior chamber into the subconjunctival space preventing damage to the optic nerve. ${ }^{1}$ The long term success rate of trabeculectomy in controlling IOP ranges from $48 \%$ to $98 \% .^{2,3}$ Owing to the complications related to the early post-operative intraocular pressure control with full thickness glaucoma surgeries, non-penetrating filtration surgery was developed. ${ }^{4}$ The designing of the sclera flaps of partial thickness over inner sclerostomy has helped to improve the outflow of aqueous humor with better 
intraocular pressure outcome ensuing decreased chances of developing hypotony and its consequences such as shallowing of the anterior chamber depth, collapse of the blood aqueous barrier, bleeding in the anterior chamber, choroidal effusion, hypotony maculopathy, formation of cataract, suprachoroidal haemorrhage and misdirection of the aqueous humor during the first few days after surgery. ${ }^{5,6}$ The designing of the original scleral flap in trabeculectomy comprised of formation of a rectangular shaped partial thickness scleral flap along with the excision of an elongated block of trabecular tissue, which has since then been modified accordingly in an effort to preserve a sufficient level of intraocular pressure in the long run and prevent the occurrence of complications. ${ }^{7,8}$ The modifications over time have included limbal or fornix-based conjunctival flaps, adjuvant anti metabolite therapy, change in shape and size of the scleral flap and inner sclerostomy as well as the level of tightness in approximation of scleral flap closure with the help of sutures, which can be adjusted or lysed and help affirm a consistent management of aqueous flow thereby reducing the risk of early post operative hypotony. ${ }^{9,10}$ Apart from all the aforementioned modifications to help maintain an adequate intraocular pressure over a longer duration of time, IOP control is mainly dependent upon the subconjunctival healing response as well. ${ }^{11,12}$ Despite the presence of various modifications, few studies have been conducted which compare the effectiveness and superiority between scleral flaps of different shapes. $^{7,8,13}$

We carried out this study to compare the outcomes of trabeculectomy with two different types of flaps, in terms of intraocular pressure management and anterior chamber depth.

\section{METHODS}

This study was carried out at Khalid eye clinic, Karachi from July 2018 to June 2019 and included 24 patients of either gender with an age range of 40 to 65 years. Patients with a diagnosis of primary open angle glaucoma and refractory to medical therapy were included in this study. Surgical procedure was explained to the patients and informed consent was obtained. Study was approved from the Ethical Review Committee. Data was collected and documented including demographic information, comorbidities, duration of glaucoma, eyelid opening, presence of eyelid deformity and current medications used. Patients who underwent previous ocular surgery and secondary glaucoma were excluded from the study. Patients were divided into two groups. Group A underwent trabeculectomy with rectangular scleral flaps whereas Group B underwent trabeculectomy with triangular scleral flap. Rest of the technique was similar in both groups. Surgery was done under general anaesthesia. After ensuring a stable IOP, limbal-based incision was made in all the patients. The overlying conjunctiva and episcleral tissue was removed from one quadrant and site cleared for the procedure. Partial thickness scleral incision in a rectangular fashion (4 $43 \mathrm{~mm}$ with $3 \mathrm{~mm}$ base) was made in group A and triangular flap ( $3 \mathrm{~mm}$ each side) was fashioned in group $B$ behind the limbus tangentially with the help of Baever blade. The anterior chamber was entered by making a central slit in the peripheral cornea using a blade under the base of the scleral flap. An elongated trabecular tissue block was removed and peripheral iridectomy was performed. Sutures were placed to approximate the scleral flaps with the rest of the sclera to make sure that the scleral flap was well positioned and sitting symmetrically within its bed. In group A, two sutures were placed diagonally through each corner of the rectangular flap. In group B, two sutures were placed through each side of the triangle close to the apex. The conjunctiva was sutured back over the scleral flap making sure no free space remained between the adjacent sutures. All surgeries were done by a single surgeon. Patients were followed for a period of up to two months. It was made sure that scleral flap was between one-half and two-thirds thickness in its entirety, sclerostomy was placed with a diameter of no greater than $1 \mathrm{~mm}$ and scleral flap was positioned with each corner well placed into the corresponding corner of the scleral bed with equal tightness of the sutures in all the surgeries. The main outcome measures were intraocular pressure reduction, anterior chamber depth and post-operative complications.

\section{RESULTS}

This study included 24 patients distributed into two groups. Each group contained twelve patients. There were fifteen $(62.5 \%)$ males and nine $(37.5 \%)$ females. Age ranged from 40 to 65 years with a mean age of $52.1 \pm 6.72$ years. There was involvement of the right eye in seventeen $(70.8 \%)$ individuals and left eye in seven $(29.2 \%)$ patients. Pre-operative mean intraocular pressure in patients of group A was $24.4 \pm 2.13$ 
$\mathrm{mmHg}$, whereas in group B it was $23.5 \pm 1.64 \mathrm{mmHg}$ (p-value 0.238 between two groups by Independent sample $\mathrm{T}$ test). IOP dropped down to $11.6 \pm 1.51$ $\mathrm{mmHg}$ in group A and $13.4 \pm 1.67 \mathrm{mmHg}$ in group B (p-value 0.11). Anterior chamber depth (ACD) was grade 4 (Von Herrick method) in patients of both groups pre-operatively. Post-operatively it remained the same in all patients of group A but two patients of group B changed to grade 3 . As far as complications were concerned, none of the patients of group A developed any post-operative hypotony, infection, or bleb leakage, where as two patients of group B developed hypotony on the first post-operative day. They did not require revision suturing and were managed conservatively. Mean follow up period was $59 \pm 2$ days post operatively.

\section{DISCUSSION}

In trabeculectomy, presence of scleral flap poses a significant resistance to the outflow of aqueous humor in the initial period thereby making scleral flap a key controller of IOP and influencing the risk of hypotony. If it is inappropriately constructed, it may cause a decreased reduction in IOP. ${ }^{14}$ The outflow of aqueous humor through a scleral flap depends upon multiple factors such as the formation of scleral tunnel, approximation of the scleral flap to the underlying scleral bed and its ability to lift to aid in drainage which depends on its elasticity as predicted by the scleral flap thickness, tension, position and tightness of the sutures placed. ${ }^{15,16}$ Keeping this in mind, this study was designed to observe the outcomes of two different shaped scleral flaps (rectangular versus triangular) and their effect on post operative intraocular pressure and anterior chamber depth. Scleral flaps of adequate size and thickness were created, since too thick or too thin flaps would result in resistance to aqueous outflow and flap dehiscence leading to uncontrolled IOP. ${ }^{17,18}$

A study was conducted in which rectangular shaped scleral flaps of $3 \times 2 \mathrm{~mm}$ were created. ${ }^{8}$ The average IOP reduction was at least two-thirds of baseline. This study also showed that the size of sclerostomy did not increase the possibility of postoperative flat anterior chamber nor did it affect the long-term IOP control. ${ }^{8}$ Another study assessed the outflow of aqueous humor in relation to the formation of scleral flap and sclerostomy made during trabeculectomy. ${ }^{19}$ Increasing the size of the scleral flap was linked to an increase in aqueous outflow. It also found that square shaped scleral flap augmented the drainage of aqueous humor as compared to triangular shaped scleral flap of same size. It also concluded that thinner scleral flap enhanced the outflow of aqueous humor but thinner than half the thickness resulted in hypotony. ${ }^{19}$ In another study, the pressure drop was greater across thinner flaps owing to less rigidity and resistance. ${ }^{7}$ A bigger scleral flap and lesser number of sutures led to a greater reduction in intraocular pressure. A comparison between rectangular and triangular shaped scleral flap was done, similar to this study, over a period of 12 months among patients of ages 40 to 76 years old. ${ }^{20}$ Patients with angle closure glaucoma were included. It was reported that the IOP reduction was equally significant among both the techniques with hypotony being found in two patients who underwent triangular shaped scleral flap and one patient who underwent rectangular shaped scleral flap, which resolved on its own within a week. The findings of the aforementioned studies correlated with the observations made in our study with the exception that patients of primary open angle glaucoma were recruited in this study and categorized into two groups. However, the reduction in IOP among both the groups was significant and rectangular technique turned out to be more effective in the significant reduction of IOP and maintenance of anterior chamber although not statistically significant. The follow up period in our study was two months as compared to the longer duration in the previous studies. The patients of group B observed a shallowing of anterior chamber depth, which could be due to the triangular design or inaccurate thickness of the scleral flap leading to increased outflow. The hypotony was managed conservatively and none of the patients required any revision surgery.

The limitation of this study is the small sample and the follow up was of short duration. Longer follow up to see the long-term effects of both shapes of flaps is needed.

\section{CONCLUSION}

Rectangular shaped scleral flap reduced IOP more as compared to triangular shaped scleral flap but the difference was not statistically significant. Patients who underwent trabeculectomy with rectangular shaped scleral flap did not show any change in the anterior chamber depth nor developed any postoperative complications. 


\section{Ethical Approval}

The study was approved by the Institutional review board/Ethical review board (ERC-26-20).

\section{Conflict of Interest}

Authors declared no conflict of interest.

\section{REFERENCES}

1. Kirwan JF, Lockwood AJ, Shah P, Macleod A, Broadway DC, King AJ, et al. Trabeculectomy Outcomes Group Audit Study Group. Trabeculectomy in the 21st century: a multicenter analysis. Ophthalmology, 2013; 120 (12): 2532-2539.

Doi: 10.1016/j.ophtha.2013.07.049.

2. Yuasa Y, Sugimoto $\mathbf{Y}$, Hirooka $\mathbf{K}$, Ohkubo $\mathbf{S}$, Higashide T, Sugiyama K, et al. Effectiveness of trabeculectomy with mitomycin $\mathrm{C}$ for glaucomatous eyes with low intraocular pressure on treatment eye drops. Acta Ophthalmol. 2020 Feb; 98 (1): e81-e87. Doi: 10.1111/aos.14195.

3. Gedde SJ, Feuer WJ, Lim KS, Barton K, Goyal S, Ahmed IIK, et al. Primary Tube Versus Trabeculectomy Study Group. Treatment Outcomes in the Primary Tube Versus Trabeculectomy Study after 3 Years of Follow-up. Ophthalmology, 2020; 127 (3): 333-345. Doi: 10.1016/j.ophtha.2019.10.002.

4. Binibrahim IH, Bergström AK. The role of trabeculectomy in enhancing glaucoma patient's quality of life. Oman J Ophthalmol. 2017; 10 (3): 150-154.

5. Walkden A, Au L, Fenerty C. Trabeculectomy Training: Review of Current Teaching Strategies. Adv Med Educ Pract. 2020; 11: 31-36.

6. Ghazala T, Imran G, Riaz A. The effectiveness of conventional trabeculectomy in controlling intraocular pressure in our population. Pak J Ophthalmol. 2013; 29: 26-30.

7. Samsudin A, Eames I, Brocchini S, Khaw PT. The Influence of Scleral Flap Thickness, Shape, and Sutures on Intraocular Pressure (IOP) and Aqueous Humor Flow Direction in a Trabeculectomy Model. J Glaucoma, 2016; 25 (7): e704-12.

Doi: 10.1097/IJG.0000000000000360.

8. Tse KM, Lee HP, Shabana N, Loon SC, Watson PG, Thean SY. Do shapes and dimensions of scleral flap and sclerostomy influence aqueous outflow in trabeculectomy? A finite element simulation approach. Br J Ophthalmol. 2012; 96 (3): 432-437. Doi: 10.1136/bjophthalmol-2011-300228.

9. O'Brart DP, Shiew M, Edmunds B. A randomised, prospective study comparing trabeculectomy with viscocanalostomy with adjunctive antimetabolite usage for the management of open angle glaucoma uncontrolled by medical therapy. $\mathrm{Br} \mathrm{J}$ Ophthalmol. 2004; 88: 1012-1017.
10. Mahadevappa K, Prasanna N, Channabasappa RA. Trends of Various Techniques of Tubectomy: A Five Year Study in a Tertiary Institute. J Clin Diagn Res. 2016; 10 (1): QC04-QC7.

11. Sheha, Hosam. Update on Modulating Wound Healing in Trabeculectomy, 2011. Doi: 10.5772/23062.

12. Yamanaka $\mathbf{O}$, Kitano-Izutani A, Tomoyose $K$. Pathobiology of wound healing after glaucoma filtration surgery. BMC Ophthalmol. 2015; 15: 157. https://doi.org/10.1186/s12886-015-0134-8

13. Dhingra S, Eames I, Nicolle A, Taban V, Brocchini S, Khaw PT. The Effect of Scleral Flap Shape and Size on Aqueous Flow and Pressure Following Trabeculectomy: Implications from Mathematical and Physical Modelling. Invest. Ophthalmol. Vis. Sci. 2011; 52 (14): 651.

14. Megan AR, Maharaj A. A Review of Scleral Flap Shape on Trabeculectomy Outcomes. Vision PanAmerica, Pan- Am J Ophthalmol. 2016; 15: 70-74.

15. Chowdhury UR, Hann CR, Stamer WD, Fautsch MP. Aqueous Humor Outflow: Dynamics and Disease. Invest. Ophthalmol. Vis. Sci. 2015; 56 (5): 2993-3003.

16. Swaminathan SS, Oh DJ, Kang MH, Rhee DJ. Aqueous outflow: segmental and distal flow. J Cataract Refract Surg. 2014; 40 (8): 1263-1272.

17. Vijaya L, Manish $\mathbf{P}$, Ronnie $\mathbf{G}$, Shantha $\mathbf{B}$. Management of complications in glaucoma surgery. Indian J Ophthalmol. 2011; 59 (1): S131-S140. doi:10.4103/0301-4738.73689

18. Henderson HW, Ezra E, Murdoch IE. Early postoperative trabeculectomy leakage: Incidence, time course, severity, and impact on surgical outcome. Br J Ophthalmol. 2004; 88: 626-629.

19. Birchall W, Bedggood A, Wells AP. Do scleral flap dimensions influence reliability of intraocular pressure control in experimental trabeculectomy? Eye, 2007. 21: 402-407.

20. Sharma A, Adhikari S, Das H, Lavaju P, Bijay S. A randomised clinical trial comparing the outcome of trabeculectomy using triangular versus rectangular scleral flaps. Nepalese J Ophthalmol. 2010; 1 (1): 2024.

\section{Authors' Designation and Contribution}

Qirat Qurban; Consultant Ophthalmologist: Concepts, Design, Literature search, Data analysis, Statistical analysis, Manuscript preparation, Manuscript editing.

Zeeshan Kamil; Consultant Ophthalmologist: Consultant Ophthalmologist: Concepts, Design, Literature search, Data analysis, Statistical analysis, Manuscript preparation, Manuscript editing.

Khalid Mahmood; Consultant Ophthalmologist: Data acquisition, Manuscript Review. 